\section{SYNLETT Spotlight 175 \\ Phenylboronic Acid (PBA): A Versatile Chemical in Synthetic Organic Chemistry}

This feature focuses on a reagent chosen by a postgraduate, highlighting the uses and preparation of the reagent in current research
Compiled by Zhi-Jian Bao

Zhi-Jian Bao was born November 4, 1981 at Suzhou, Jiangsu Province (P. R. of China). He received his BSc from Jiangsu Institute of Education. Presently he is working as a postgraduate at Suzhou University, under the supervision of Professor Shun-Jun Ji. His field of research is ionic liquids.

College of Chemistry and Chemical Engineering of Suzhou University, Suzhou 215123, P. R. of China

E-mail: baozjian@sohu.com

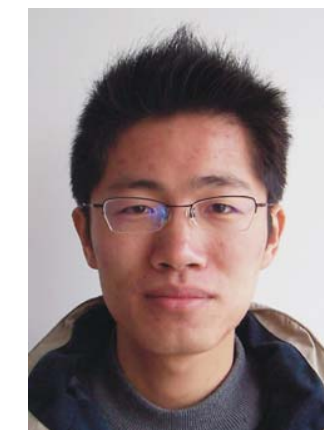

hydroxyl groups, ${ }^{6}$ addition of PBA to $\alpha, \beta$-unsaturated ketones, ${ }^{7,8} \mathrm{~N}$ - and O-arylations, ${ }^{9}$ and a catalytic triple condensation reaction. ${ }^{10}$ In addition, PBA is also used in carbohydrate sensor design. ${ }^{12-14}$
Recently phenylboronic acid (PBA) has been used in organic synthesis as a versatile reagent. Examples are Suzuki reactions,,${ }^{1-3}$ the coupling of PBA with styrene, ${ }^{4}$ a ring-opening reaction of epoxy sulfide, ${ }^{5}$ the protection of
(B) Amengual et al. first turned our attention to the coupling of boronic acids with either styrene or 2-vinylpyridine. ${ }^{4}$ The addition of various boronic acids to styrene and 2-vinylpyridine was realized with high selectivity and yield.

(A) Suzuki couplings $s^{1,2}$ of indoles ${ }^{3}$ with phenylboronic acids gave the products in high yields, which were unaffected by Boc or Tos protection at the heterocyclic nitrogen.
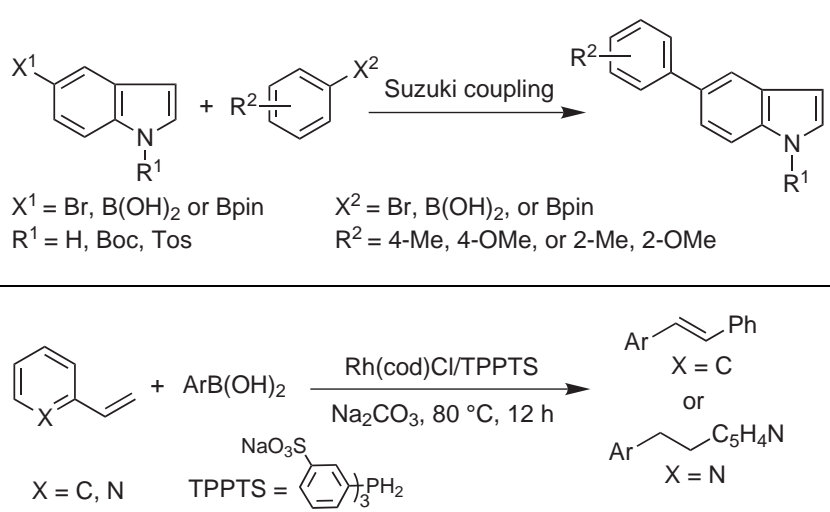

(C) PBA is used in the ring-opening reaction of epoxy sulfides in benzene, which has been demonstrated to occur stereospecifically via episulfonium ions, i.e, with double inversion of the configuration, giving rise to phenylboronic esters of 2,3-diols ${ }^{5}$ in quantitative yields.

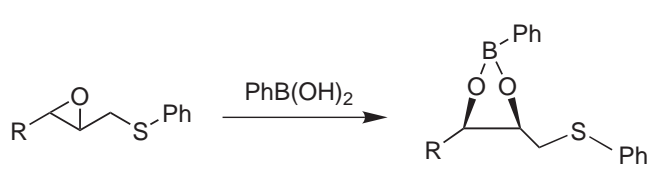

(D) Yan et al. ${ }^{6}$ reported on the development of an arylboronic acid as protecting group for diols. Both protection and deprotection can be accomplished under mild conditions with quantitative conversion (yield: 91-95\%).

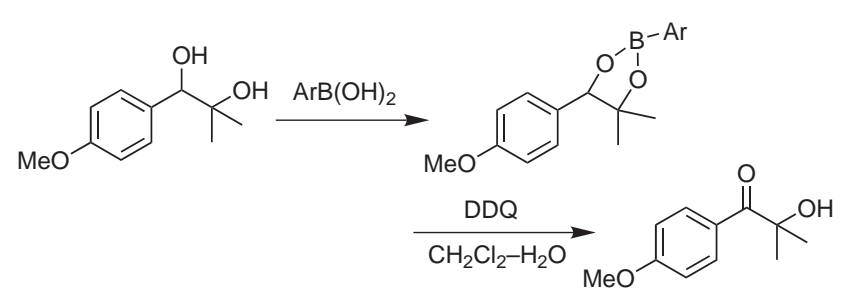

SYNLETT 2006, No. 16, pp 2679-2680

Advanced online publication: 22.09.2006

DOI: 10.1055/s-2006-951472; Art ID: V17906ST

(c) Georg Thieme Verlag Stuttgart · New York 
(E) Reaction of phenylboronic acid with 2-cyclohexenone in the presence of $3 \mathrm{~mol} \%$ of a rhodium $(\mathrm{I}) /(S)$-Binap catalyst in dioxane$\mathrm{H}_{2} \mathrm{O}(10: 1)$ at $100{ }^{\circ} \mathrm{C}$ proceeded with high enantioselectivity to give $(S)$-3-phenylcyclohexanone in high yield and with up to $99 \%$ ee. $^{7}$

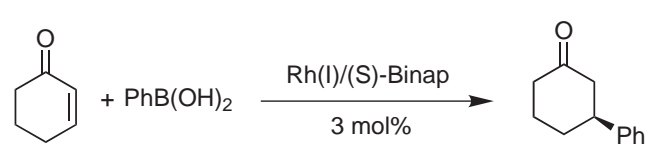

(F) In addition, PBA can be applied in the asymmetric 1,4-addition to $\alpha, \beta$-unsaturated amides. ${ }^{8}$

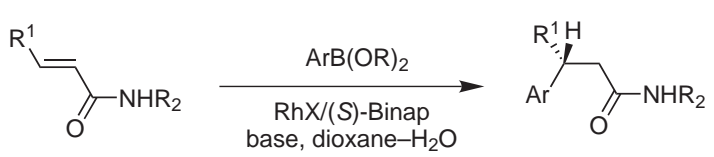

(G) A new method of arylating NH- and OH-containing compounds at room temperature with PBA and copper(II) acetate in the presence of a tertiary amine promoter was described. Substrates include phenols, amines, anilines, amides, imides, ureas, carbamates, and sulfonamides. ${ }^{9}$

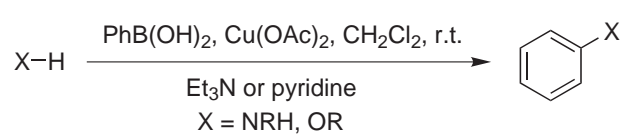

(H) A remarkable phenylboronic acid mediated triple condensation reaction of phloroglucinol with a series of $\alpha, \beta$-unsaturated carbonyl compounds was reported. ${ }^{10}$ These derivatives represent structural analogues of the natural product xyloketal A, which was reported to be a potent inhibitor of acetylcholine esterase.

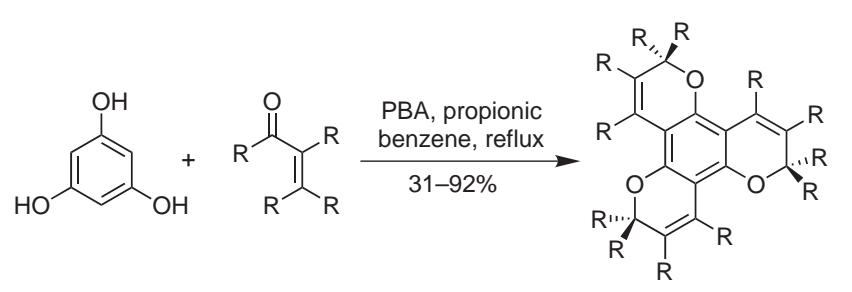

(I) The palladium-catalyzed tandem reactions of yne-propargylic carbonates with aryl boronic acids, followed by $6 \pi$-electrocyclization to give fused-ring aromatic products ${ }^{11}$ such as naphthalene, benzofuran, and benzothiophene derivatives were realized.

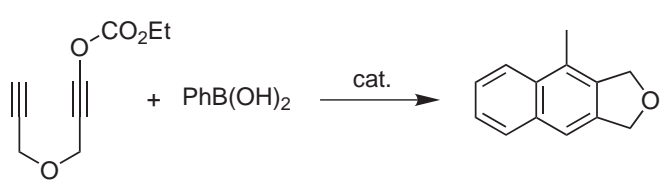

(J) PBA is also used in carbohydrate sensor design. ${ }^{12,13}$ The formation of highly stable phenylboronate esters of the $\beta$-furanose form of this compound was reported. The $\beta$-furanose anomer is selectively recognized by phenylboronate. ${ }^{14}$

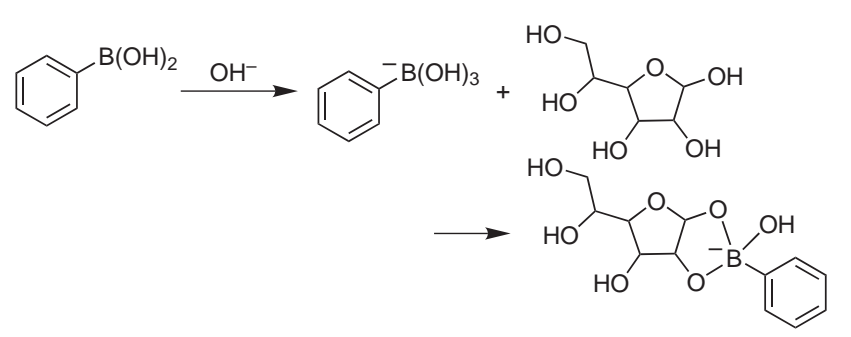

\section{References}

(1) Dai, W.-M.; Li, Y.; Zhang, Y.; Lai, K. W.; Wu, J. Tetrahedron Lett. 2004, 45, 1999.

(2) Leadbeater, N. E.; Marco, M. J. Org. Chem. 2003, 68, 888.

(3) Prieto, M.; Zurita, E.; Rosa, E.; Munoz, L.; Lloyd-Williams, P.; Giralt, E. J. Org. Chem. 2004, 69, 6812.

(4) Amengual, R.; Michelet, V.; Genêt, J.-P. Tetrahedron Lett. 2002, 43, 5905.

(5) Hayakawa, H.; Okada, N.; Miyashita, M. Tetrahedron Lett. 1999, 40, 3191

(6) Yan, J.; Jin, S.; Wang, B.-H. Tetrahedron Lett. 2005, 46, 8503.

(7) Yoshiaki, T.; Masamichi, O.; Tamio, H. Chirality 2000, 12, 469.
(8) Satoshi, S.; Norio, M. J. Org. Chem. 2001, 66, 8944.

(9) Chan, D.-M.-T.; Monaco, K.-L.; Wang, R.-P.; Winters, M.P. Tetrahedron Lett. 1998, 39, 2933.

(10) Pettigrew, J. D.; Cadieux, J. A.; So, S. S. S.; Wilsch, P. D. Org. Lett. 2005, 7, 467.

(11) Wang, F.; Tong, X.-F.; Cheng, J.; Zhang, Z.-G. Chem. Eur. J. 2004, 10, 5338 .

(12) Otsuka, H.; Uchimura, E.; Koshino, H.; Okano, T.; Kataoka, K. J. Am. Chem. Soc. 2003, 125, 3493.

(13) Dicesare, N.; Lakowicz, J.-R. Tetrahedron Lett. 2001, 42, 9105.

(14) Rohovec, J.; Maschmeyer, T.; Aime, S.; Peters, J. A. Chem. Eur. J. 2003, 9, 2193. 